UWO-TH-09/4

\title{
Relativistic Conformal Magneto-Hydrodynamics from Holography
}

\author{
Evgeny I. Buchbinder ${ }^{1}$, Alex Buchel ${ }^{1,2}$ \\ ${ }^{1}$ Perimeter Institute for Theoretical Physics \\ Waterloo, Ontario N2J 2W9, Canada \\ ${ }^{2}$ Department of Applied Mathematics \\ University of Western Ontario \\ London, Ontario N6A 5B\%, Canada
}

\begin{abstract}
We use the AdS/CFT correspondence to study first-order relativistic viscous magnetohydrodynamics of $(2+1)$ dimensional conformal magnetic fluids. It is shown that the first order magneto-hydrodynamics constructed following Landau and Lifshitz from the positivity of the entropy production is inconsistent. We propose additional contributions to the entropy motivated dissipative current and, correspondingly, new dissipative transport coefficients. We use the strongly coupled M2-brane plasma in external magnetic field to show that the new magneto-hydrodynamics leads to self-consistent results in the shear and sound wave channels.
\end{abstract}

November 3, 2018 
A theory of viscous magneto-hydrodynamics [1] has a wide range of practical applications in condensed matter [2] (and references therein) and astrophysics [3] (and references therein). It is best thought as an effective low-energy theory organizing derivative expansion of systems in local, but not global, equilibrium. For concreteness, consider relativistic $d$ dimensional charged fluid in external electro-magnetic field $F^{\mu \nu}$. The conserved quantities associated with the system are the stress-energy tensor $T^{\mu \nu}$ and the electric current $d$-vector $J^{\mu}$. The dynamics of the hydrodynamic fluctuations in the fluid is governed by the following equations of motion

$$
\partial_{\mu} J^{\mu}=0, \quad \partial_{\nu} T^{\mu \nu}=F^{\mu \nu} J_{\nu},
$$

where the total current and the stress-energy tensor include the equilibrium part (with local energy density $\epsilon$, pressure $P$, and charge density $\rho)$ and the dissipative parts $\left(\nu_{\mu}\right.$ and $\Pi^{\mu \nu}$ correspondingly):

$$
T^{\mu \nu}=\epsilon u^{\mu} u^{\nu}+P \Delta^{\mu \nu}+\Pi^{\mu \nu}, \quad J^{\mu}=\rho u^{\mu}+\nu^{\mu} .
$$

In Eq. (2)

$$
\Delta^{\mu \nu}=\eta^{\mu \nu}+u^{\mu} u^{\nu}, \quad \Pi^{\mu}{ }_{\nu} u^{\nu}=0, \quad u^{\mu} \nu_{\mu}=0,
$$

where $u^{\mu}$ is the local $d$-velocity of the fluid, $u^{\mu} u_{\mu}=-1$. In phenomenological hydrodynamics one represents the dissipative terms $\nu_{\mu}$ and $\Pi^{\mu \nu}$ as an infinite series expansion in velocity gradients, with coefficients of the expansion commonly referred to as transport coefficients. A familiar example are the (neutral) fluid Navier-Stokes equations, obtained by truncating the dissipative stress-energy tensor $\Pi^{\mu \nu}$ to linear order in the velocity gradients

$$
\Pi^{\mu \nu}=-\eta \sigma^{\mu \nu}-\zeta \Delta^{\mu \nu}\left(\partial_{\alpha} u^{\alpha}\right),
$$

where

$$
\sigma^{\mu \nu}=\Delta^{\mu \alpha} \Delta^{\nu \beta}\left(\partial_{\alpha} u_{\beta}+\partial_{\beta} u_{\alpha}\right)-\frac{2}{d-1} \Delta^{\mu \nu} \Delta^{\alpha \beta}\left(\partial_{\gamma} u^{\gamma}\right) .
$$

Notice that at this order in the hydrodynamic approximation we need to introduce only two transport coefficients: the shear $\eta$ and bulk $\zeta$ viscosities. Higher orders in the velocity gradients expansion for $\Pi^{\mu \nu}$, and for charged fluids the dissipative current velocity gradients expansion, necessitate introduction of additional transport coefficients. It is desirable to formulate a principle that would constrain such a rapidly growing set 
of transport coefficients. A popular approach, due to Landau and Lifshitz [1], is to define an off-equilibrium entropy current and constrain transport coefficients by imposing the positivity of its divergence (equivalently, the positivity of the entropy production in relaxation of a near-equilibrium state). In this framework the authors of [2] (HKMS) determined the first-order in $d$-velocity gradients expression for the dissipative current $\nu_{\mu}:$

$$
\nu^{\mu}=\sigma_{Q} \Delta^{\mu \nu}\left(-\partial_{\nu} \mu+F_{\nu \alpha} u^{\alpha}+\frac{\mu}{T} \partial_{\nu} T\right)
$$

where $T$ and $\mu$ and the local temperature and the chemical potential. Remarkably, only a single new transport coefficient (in addition to $\{\eta, \zeta\}$ ), a conductivity $\sigma_{Q}$, is needed to describe first-order HKMS magneto-hydrodynamics. It is important to stress that in [2], the authors considered the case of the electro-magnetic field vanishingly small in the hydrodynamic limit, that is $F \sim \mathcal{O}(\partial u)$.

The question that we raise in this Letter is whether the principle of the positivity of the entropy production leads to a consistent theory of magneto-hydrodynamics for an arbitrary external electro-magnetic field, not necessarily small in the hydrodynamic limit. Unless there is a first principle microscopic derivation of hydrodynamics, it is impossible to address such a question. In this Letter we consider the three-dimensional maximally supersymmetric Yang-Mills theory with large number of colors at the infrared fixed point. This theory is commonly referred to as the theory of M2-branes. Then we use AdS/CFT correspondence [4] of the strongly coupled M2-brane plasma in external magnetic field to argue that the first-order dissipative current $\nu_{\mu}$ has to be modified as follows

$$
\begin{aligned}
\nu^{\mu} & =\sigma_{Q} \Delta^{\mu \nu}\left(-\partial_{\nu} \mu+F_{\nu \alpha} u^{\alpha}+\frac{\mu}{T} \partial_{\nu} T\right) \\
& -\hat{\sigma}_{Q} \Delta^{\mu \nu} F_{\nu \alpha} u^{\gamma} \partial_{\gamma}\left(\frac{u^{\alpha}}{T}\right)
\end{aligned}
$$

where $\hat{\sigma}_{Q}$ is the new conductivity transport coefficient. Given that AdS/CFT correspondence provides a microscopic derivation of the M2-brane hydrodynamics, we compute that in this particular case and at zero charge density at equilibrium

$$
\hat{\sigma}_{Q}=\frac{3}{2 \pi} \sigma_{Q}
$$

This implies that the principle of the positivity of the entropy production is violated in magneto-hydrodynamics already at the linearized level as the theory of M2-branes represents an explicit counter-example. On the contrary, in neutral hydrodynamics 
with no external fields this principle holds to linear order and is violated at second order [5].

Our approach to introducing the transport coefficients to describe the dissipative current $\nu^{\mu}$ in conformal magneto-hydrodynamics will be based on conformal invariance. A similar approach to conformal hydrodynamics with no extra charges and fields was undertaken in [5]. Under the Weyl rescaling with a local parameter $\Omega$ we have the following transformation properties

$$
\begin{aligned}
& T^{\mu \nu} \rightarrow e^{(d+2) \Omega} T^{\mu \nu}, \quad J^{\mu} \rightarrow e^{d \Omega} J^{\mu}, \quad F_{\mu \nu} \rightarrow F_{\mu \nu}, \\
& \epsilon \rightarrow e^{d \Omega} \epsilon, \quad T \rightarrow e^{\Omega} T, \quad u^{\mu} \rightarrow e^{\Omega} u^{\mu}, \quad \mu \rightarrow e^{\Omega} \mu .
\end{aligned}
$$

These properties are obtained from demanding that eqs. (1) are Weyl invariant. Based on the principle of conformal invariance as well as transversality $u_{\mu} \nu^{\mu}=0$, we propose the following expression for the dissipative part of the current up to terms which do not contribute to linear order in fluctuations. We get

$$
\begin{aligned}
\nu^{\mu}= & \sigma_{Q 1} \Delta^{\mu \nu}\left(\frac{\mu}{T} \partial_{\nu} T-\partial_{\nu} \mu\right)+\sigma_{Q 2} \Delta^{\mu \nu} F_{\nu \alpha} u^{\alpha} \\
& -\hat{\sigma}_{Q} \Delta^{\mu \nu} F_{\nu \alpha} u^{\gamma} \partial_{\gamma}\left(\frac{u^{\alpha}}{T}\right) .
\end{aligned}
$$

We have introduced the two different coefficients $\sigma_{Q 1}$ and $\sigma_{Q 2}$ instead of $\sigma_{Q}$ since the first two terms individually are conformally invariant.

Let us discuss whether there are possible corrections to (10). Our discussion will be rather brief since these extra terms will not play any role in the rest of the Letter. First, there is one more term linear in $F$ which we can add in (10), namely

$$
\tilde{\sigma}_{Q} \Delta^{\mu \nu} F_{\nu \alpha}\left(\frac{\mu}{T} \partial^{\alpha} T-\partial^{\alpha} \mu\right)
$$

In the rest of the paper, we will consider the neutral M2-brane plasma and set $\rho=$ 0. In this case, as we will see below, there is a certain decoupling of the linearized hydrodynamic equations. The term (11) is inconsistent with the decoupling and, hence, $\tilde{\sigma}_{Q}=0$ in this particular case. Note that, in general, $\tilde{\sigma}_{Q}=0$ does not have to vanish and the term (11) can appear in (10). However, it vanishes for the theory under study. Similarly, one can analyze terms with higher powers of $F_{\mu \nu}$. It is not difficult to show that such terms either are inconsistent with the decoupling (so the coefficient in front of them has to vanish just like $\tilde{\sigma}_{Q}=0$ ) or can be used to redefine the transport coefficients in (10) at higher order of $F^{2}$. The latter is due to the fact that in pure 
magnetic background in three dimensions $F_{\mu}^{\alpha} F_{\alpha \nu} \sim F^{2} \eta_{\mu \nu}$. Thus, we would like to stress that our proposal in eq. (11) is not the most general expansion of the current in the external electro-magnetic field. As we just explained there can be additional terms invisible by the magnetized M2-brane theory plasma. The purpose of this Letter is not to develop such an expansion but rather to explicitly demonstrate that the principle of the positivity of the entropy production in violated in magneto-hydrodynamics at the linearized level.

We would like now to test our phenomenological proposal of first order magnetohydrodynamics in the framework of soluble AdS/CFT correspondence [4]. Specifically, we consider magneto-hydrodynamics of dyonic black holes in $A d S_{4} \times S^{7}$ supergravity of M-theory. The latter realizes a holographic dual of the strongly coupled conformal $(2+1)$ dimensional magnetized plasma. The equilibrium state of the plasma is described by a dyonic black hole in the effective four-dimensional Einstein-Maxwell gravity with a negative cosmological constant [6]. A thermodynamic potential of the plasma is given by

$$
\Omega=-V_{2} p=V_{2} \frac{1}{g^{2}} \frac{\alpha^{3}}{4}\left(-1-\frac{\mu^{2}}{\alpha^{2}}+3 \frac{B^{2}}{\alpha^{4}}\right)
$$

where $V_{2}$ is the spatial area and $p$ is the thermodynamic pressure. Furthermore, $g^{2}$ is the bulk gravitational coupling, related to the central charge $c$ of the M2-brane CFT as $c=96 g^{-2}$ (it can be computed from eq. (5) in [7]), $\mu$ is the electric charge chemical potential, and $B$ is an external magnetic field. The parameter $\alpha$ entering (12) is related to the temperature $T$ as follows

$$
\frac{4 \pi T}{\alpha}=3-\frac{\mu^{2}}{\alpha^{2}}-\frac{B^{2}}{\alpha^{4}}
$$

and is introduced to simplify expressions for further thermodynamic quantities. For example, the equilibrium energy density $\epsilon$, the entropy density $s$, the charge density $\rho$ and the magnetization $M$ per unit areas are given by

$$
\begin{aligned}
& \epsilon=\frac{1}{g^{2}} \frac{\alpha^{3}}{2}\left(1+\frac{\mu^{2}}{\alpha^{2}}+\frac{B^{2}}{\alpha^{4}}\right), \quad s=\frac{1}{g^{2}} \alpha^{2}, \\
& \rho=\frac{1}{g^{2}} \alpha \mu, \quad M=-\frac{1}{g^{2}} \frac{B}{\alpha} .
\end{aligned}
$$

Furthermore, the external magnetic field $B$ naturally enters the supergravity analysis via the parameter $h=\frac{B}{\alpha^{2}}$.

We denote $\left(x^{0}=t, x^{1}=x, x^{2}=y\right)$ and refer to nonzero components of the electromagnetic field strength as $F_{x y}=-F_{y x}=B$. Eq. (10) for the dissipative current 
together with eqs. (1)-(5) is our starting point. Before we discuss the linearized equations of motion, let us point out that the appearance of the magnetic field substantially increases the possible set of the hydrodynamic regimes which one can study. That is, instead of taking $B$ to be fixed in the hydrodynamic limit of small momentum $q$, we can simultaneously scale $B$ as $q^{p}, p>0$. As explained in [8, 9], the new hydrodynamic regimes allow us to study effects of magnetic field on the dispersion relation of the sound and the shear quasinormal modes in a controllable setting, i.e., when it is consistent to neglect the second and higher order hydrodynamic contributions, as well as nonlinear effects. In this Letter, we will study the cases of fixed $B$ and $B \sim q^{1 / 2}$. This will allow us to extract the coefficients $\sigma_{Q 1}, \sigma_{Q 2}$ and $\hat{\sigma}_{Q}$ for the M2-brane plasma.

We expand eqs. (1) around the equilibrium state $u^{\mu}=(1,0,0), T=$ const, $\mu=0$ to linear order in fluctuations. As usual, the fluctuations are of the plane wave form $e^{-i \omega t+i q y}$. As the set of independent fluctuations we can choose $\left(\delta \epsilon, \delta \mu, \delta u_{x}, \delta u_{y}\right)$. In deriving the equations of motion, in addition to setting $\rho=0, \mu=0$, we will also set (see eqs. (14))

$$
\left(\frac{\partial \rho}{\partial T}\right)_{\mu}=0, \quad\left(\frac{\partial \epsilon}{\partial \mu}\right)_{T}=0, \quad\left(\frac{\partial \rho}{\partial \mu}\right)_{T}=\frac{1}{g^{2}} \alpha .
$$

At $\rho=0$, the equations of motion decouple into the two separate pairs. The first pair reads

$$
\begin{aligned}
& 0=\omega \delta \epsilon-\frac{3}{2} q \epsilon \delta u_{y} \\
& 0=\frac{3}{2} \omega \epsilon \delta u_{y}-\frac{1}{2} q \delta \epsilon+i q^{2} \eta \delta u_{y}+i \sigma_{Q 2} B^{2} \delta u_{y} \\
& -\frac{\omega \hat{\sigma}_{Q} B^{2} \delta u_{y}}{T}
\end{aligned}
$$

where we have used the equation of state $P=\epsilon / 2$ followed from conformal invariance. When $B=0$ these two equations describe sound waves and we will refer to them as to the "sound channel". The second pair of equations is

$$
\begin{aligned}
& 0=\frac{3}{2} \omega \epsilon \delta u_{x}-q B \sigma_{Q 1} \delta \mu+i \sigma_{Q 2} B^{2} \delta u_{x}+i q^{2} \eta \delta u_{x} \\
& -\frac{\omega \hat{\sigma}_{Q} B^{2} \delta u_{x}}{T} \\
& 0=\omega\left(\frac{\partial \rho}{\partial \mu}\right)_{T} \delta \mu+q \sigma_{Q 2} B \delta u_{x}+i q^{2} \sigma_{Q 1} \delta \mu \\
& +\frac{i \omega q \hat{\sigma}_{Q} B \delta u_{x}}{T} .
\end{aligned}
$$


When $B=0$ these two equations further decouple. One of them describes a shear mode and the other one describes charge diffusion. We will refer to this pair as to the "shear channel". The decoupling between the sound and the shear channels was also observed in the supergravity description of the M2-brane plasma with vanishing equilibrium charge density [8]. Now note that the term (11) would lead to the mixing between the sound and shear channels. Hence, $\tilde{\sigma}_{Q}=0$ at least at $\rho=0$. Unfortunately, at this point, we are not able to say whether the term (11) is present in general in magneto-hydrodynamics.

Let us now discuss solutions to eqs. (16) and (17) in the regimes specified above. Recall that in the context of AdS/CFT correspondence hydrodynamic fluctuations in plasma, i.e., the sound modes and the shear modes, are related to the lowest quasinormal modes of the black hole solutions dual to the equilibrium state of the plasma [10]. For the magnetized M2-brane plasma of interest here, these quasinormal modes were extensively studied in $[8,9]$. For convenience, we introduce $\mathfrak{w}=\omega /(2 \pi T)$ and $\mathfrak{q}=|\vec{q}| /(2 \pi T)$.

Hydrodynamic limit with $B$ held fixed. In the sound channel we obtain a constant cyclotron mode and a diffusive mode

$$
\omega_{C F T}^{\text {sound }}=-i q^{2} \frac{3 \epsilon}{4 \sigma_{Q 2} B^{2}} .
$$

This mode was reproduced on the supergravity side in [9] where the comparison has also been made. The result of this comparison is that

$$
\sigma_{Q 2}=\frac{1}{g^{2}}
$$

It is important to stress that this result is exact to all orders in magnetic field. In the shear channel we obtain a cyclotron mode and a subdiffusive mode

$$
\omega_{C F T}^{\text {shear }}=-i q^{4} \frac{\eta}{B^{2}\left(\frac{\partial \rho}{\partial \mu}\right)_{T}} \frac{\sigma_{Q 1}}{\sigma_{Q 2}} .
$$

This mode was reproduced on the supergravity side in [9]. Moreover, in [9] $\eta$ was found from a Kubo formula to all orders in magnetic field to satisfy

$$
\frac{\eta}{s}=\frac{1}{4 \pi} \text {. }
$$

It was further generalized for the case of $\rho \neq 0$ in [11]. Comparison with the supergravity result of $[9]$ then implies that $\sigma_{Q 1}=\sigma_{Q 2}$. From now on we will remove the 
subscripts "1" and "2" and denote $\sigma_{Q 1}=\sigma_{Q 2}=\sigma_{Q}$. Strictly speaking we find that $\sigma_{Q 1}=\sigma_{Q 2}$ only for the M2-brane plasma. However, it is possible that this is true in general.

Hydrodynamic limit with $H \equiv \frac{h}{\sqrt{\mathfrak{q}}}$ held fixed. The relevant dispersion relation for the sound channel quasinormal mode of dyonic black hole describing strongly coupled M2brane plasma was computed in [8]:

$$
\begin{aligned}
& \mathfrak{w}_{\text {SUGRA }}^{\text {sound }}=\Gamma_{0}^{\text {sound }} \mathfrak{q}+i \Gamma_{1}^{\text {sound }} \mathfrak{q}^{2}+\mathcal{O}\left(\mathfrak{q}^{3}\right) \\
& \Gamma_{0}^{\text {sound }}=-i \frac{4}{9} H^{2} \pm \frac{1}{\sqrt{2}} \sqrt{1-\frac{32}{81} H^{4}} \\
& \Gamma_{1}^{\text {sound }}=-\frac{1}{4}-\frac{8}{9} H^{4} \pm \frac{2 i H^{2}\left(32 H^{4}-45\right)}{9 \sqrt{162-64 H^{4}}}
\end{aligned}
$$

On the CFT side, the dispersion relation in the sound channel now depends on the new coefficient $\hat{\sigma}_{Q}$ :

$$
\begin{aligned}
& \mathfrak{w}_{C F T}^{\text {sound }}=\Gamma_{0}^{\text {sound }} \mathfrak{q}+i \Gamma_{1}^{\text {sound }} \mathfrak{q}^{2} \\
& +H^{2}\left(\frac{32}{27} H^{2} \mp \frac{4 i\left(64 H^{4}-81\right)}{27 \sqrt{162-64 H^{4}}}\right)\left(1-\frac{2 \pi}{3} \frac{\hat{\sigma}_{Q}}{\sigma_{Q}}\right) \mathfrak{q}^{2} \\
& +\mathcal{O}\left(\mathfrak{q}^{3}\right)
\end{aligned}
$$

where we used equilibrium thermodynamic properties of the M2-brane plasma, eqs. (14), the universality of its viscosity, eq. (21), and eq. (19). If we assume that the principle of the positivity of the entropy production holds to linear order and set $\hat{\sigma}_{Q}$ to zero we find that

$$
\mathfrak{w}_{S U G R A}^{\text {sound }}-\mathfrak{w}_{C F T}^{\text {sound }}=\mathcal{O}\left(\mathfrak{q}^{2}\right) \neq \mathcal{O}\left(\mathfrak{q}^{3}\right)
$$

This shows that the formulation of magneto-hydrodynamics based on this principle is inconsistent. Moreover, the inconsistency appears already at the linearized level. In our framework of new dissipative magneto-hydrodynamics (10) the inconsistency is resolved by simply declaring that

$$
\hat{\sigma}_{Q}=\frac{3}{2 \pi} \sigma_{Q}
$$

Note that, formally, this relation is computed only to leading order in $B^{2} / T^{4}$ since our analysis is done in the hydrodynamic limit with the vanishing magnetic field. However, given that $\sigma_{Q}$ is independent of $B^{2} / T^{4}$, it is natural to expect that so is $\hat{\sigma}_{Q}$.

Can we find an independent check on relation (25) between the two conductivities of the M2-brane plasma? Fortunately, the answer is yes! Consider the shear channel 
hydrodynamic regime with $H=\frac{h}{\sqrt{\mathfrak{q}}}$ held fixed. On the CFT side the dispersion relation again depends on the new coefficient $\hat{\sigma}_{Q}$ :

$$
\begin{aligned}
\mathfrak{w}_{C F T}^{\text {shear }}= & -i \frac{8}{9} H^{2} \mathfrak{q}+i\left(-2+\left(\frac{16}{27}-\frac{128 \pi}{81} \frac{\hat{\sigma}_{Q}}{\sigma_{Q}}\right) H^{4}\right) \mathfrak{q}^{2} \\
& +\mathcal{O}\left(\mathfrak{q}^{3}\right)
\end{aligned}
$$

where once again, we used equilibrium thermodynamic properties of the M2-brane plasma, eqs. (14), the universality of its viscosity, eq. (21), and eq. (19).

Although the shear channel analysis in [9] were not done in the hydrodynamic regime with $B \sim q^{1 / 2}$, it is straightforward to do so. Following notations of [9], the gauge-invariant wave-functions of the shear channel quasinormal modes $\left\{Z_{H}, Z_{A}\right\}$, in the hydrodynamic limit with $\frac{h}{\sqrt{\mathfrak{q}}}$ kept constant, have the following hydrodynamic expansion

$$
\begin{aligned}
Z_{H}(r)= & f(r)^{-i \mathfrak{w} / 2}\left(Z_{0}(r)+i \mathfrak{q} Z_{1}(r)+\mathfrak{q}^{2} Z_{2}(r)+\mathcal{O}\left(\mathfrak{q}^{3}\right)\right) \\
Z_{A}(r)= & f(r)^{-i \mathfrak{w} / 2} \alpha H \sqrt{\mathfrak{q}}\left(A_{0}(r)+i \mathfrak{q} A_{1}(r)+\mathfrak{q}^{2} A_{2}(r)\right. \\
& \left.+\mathcal{O}\left(\mathfrak{q}^{3}\right)\right)
\end{aligned}
$$

As in [9], imposing the incoming wave boundary conditions at the horizon and the Dirichlet conditions at the boundary for $\left\{Z_{H}, Z_{A}\right\}$ determines the dispersion relation for the hydrodynamic shear mode

$$
\begin{aligned}
& \mathfrak{w}_{\text {SUGRA }}^{\text {shear }}=\Gamma_{0}^{\text {shear }} \mathfrak{q}+i \Gamma_{1}^{\text {shear }} \mathfrak{q}^{2}+\mathcal{O}\left(\mathfrak{q}^{3}\right) \\
& \Gamma_{0}^{\text {shear }}=-i \frac{8}{9} H^{2}, \quad \Gamma_{1}^{\text {shear }}=-2-\frac{16}{9} H^{4} .
\end{aligned}
$$

Requiring that

$$
\mathfrak{w}_{S U G R A}^{\text {shear }}-\mathfrak{w}_{C F T}^{\text {shear }}=\mathcal{O}\left(\mathfrak{q}^{3}\right)
$$

we arrive at the same value for $\hat{\sigma}_{Q}$, as the one obtained in the sound channel, eq. (25).

In this Letter, we presented a new phenomenological framework of first-order viscous magneto-hydrodynamics. Using a soluble microscopic model in string theory, in which we can rigorously derive an effective hydrodynamic description, we established that the first-order magneto-hydrodynamics constructed from the positivity of the entropy current is inconsistent. A careful analysis of conformal invariance of the effective hydrodynamic description allowed us to identify additional dissipative terms. 
We showed that the modified magneto-hydrodynamics provides a consistent interpretation of AdS/CFT results. On the other hand, we point out that there is no conflict between our results and the HKMS approach [2] who considered the case of the small magnetic field in the hydrodynamic limit.

Acknowledgements. The authors would like to thank Sam Vazquez for valuable discussions. Research at Perimeter Institute is supported by the Government of Canada through Industry Canada and by the Province of Ontario through the Ministry of Research \& Innovation. AB gratefully acknowledges further support by an NSERC Discovery grant and support through the Early Researcher Award program by the Province of Ontario.

\section{References}

[1] L. D. Landau and E. M. Lifshitz, "Fluid Mechanics," Pergamon Press, 1987.

[2] S. A. Hartnoll, P. K. Kovtun, M. Muller and S. Sachdev, "Theory of the Nernst effect near quantum phase transitions in condensed matter, and in dyonic black holes," Phys. Rev. B 76, 144502 (2007) [arXiv:0706.3215 [cond-mat.str-el]].

[3] J. M. Stone and M. L. a. Norman, "ZEUS-2D: A Radiation magnetohydrodynamics code for astrophysical flows in two space dimensions. I. The Hydrodynamic algorithms and tests," Astrophys. J. Suppl. 80 (1992) 753; Astrophys. J. Suppl. 80 (1992) 791.

[4] J. M. Maldacena, "The large $N$ limit of superconformal field theories and supergravity," Adv. Theor. Math. Phys. 2, 231 (1998); Int. J. Theor. Phys. 38, 1113 (1999) [arXiv:hep-th/9711200].

[5] R. Baier, P. Romatschke, D. T. Son, A. O. Starinets and A. Stephanov, "Relativistic viscous hydrodynamics, conformal invariance, and holography," JHEP 0804, 100 (2008) [arXiv:0712.2451 [hep-th]].

[6] S. A. Hartnoll and P. Kovtun, "Hall conductivity from dyonic black holes," Phys. Rev. D 76, 066001 (2007) [arXiv:0704.1160 [hep-th]].

[7] P. Kovtun and A. Ritz, "Black holes and universality classes of critical points ," Phys.Rev.Lett.100:171606,2008 [arXiv:0801.2785 [hep-th]]. 
[8] E. I. Buchbinder, A. Buchel and S. E. Vazquez, "Sound Waves in $(2+1)$ Dimensional Holographic Magnetic Fluids," JHEP 0812, 090 (2008) [arXiv:0810.4094 [hep-th]].

[9] E. I. Buchbinder and A. Buchel, "The Fate of the Sound and Diffusion in Holographic Magnetic Field," Phys. Rev. D 79, 046006 (2009) [arXiv:0811.4325 [hepth]].

[10] D. T. Son and A. O. Starinets, "Viscosity, Black Holes, and Quantum Field Theory," Ann. Rev. Nucl. Part. Sci. 57, 95 (2007) [arXiv:0704.0240 [hep-th]].

[11] J. Hansen and P. Kraus, "Nonlinear Magnetohydrodynamics from Gravity," JHEP 0904048 (2009) [arXiv:0811.3468 [hep-th]]. 